\title{
Health Equity and Colorectal Cancer Awareness: a Community Health Educator Initiative
}

\author{
Michael A. Preston ${ }^{1}$ (i) $\cdot$ Debbie Cadet $^{1} \cdot$ Rachel Hunley $^{1} \cdot$ Reuben Retnam $^{1} \cdot$ Sarah Arezo $^{1} \cdot$ Vanessa B. Sheppard $^{1}$
}

Accepted: 9 October 2021 / Published online: 22 October 2021

(c) American Association for Cancer Education 2021

\begin{abstract}
Disparities in colorectal cancer (CRC) incidence and mortality persist in rural and underserved communities. Our Community Outreach and Engagement (COE) activities are grounded in a bi-directional Community-to-Bench model in which the National Outreach Network Community Health Educator (NON CHE) Screen to Save (S2S) initiative was implemented. In this study, we assessed the impact of the NON CHE S2S in rural and underserved communities. Descriptive and comparative analyses were used to examine the role of the NON CHE S2S on CRC knowledge and CRC screening intent. Data included demographics, current CRC knowledge, awareness, and future $\mathrm{CRC}$ health plans. A multivariate linear regression was fit to survey scores for CRC knowledge. The NON CHE S2S engaged 441 participants with 170 surveys completed. The difference in participants' CRC knowledge before and after the NON CHE S2S intervention had an overall mean of 0.92 with a standard deviation of 2.56. At baseline, White participants had significantly higher CRC knowledge scores, correctly answering $1.94(p=0.007)$ more questions on average than Black participants. After the NON CHE S2S intervention, this difference was not statistically significant. Greater than $95 \%$ of participants agreed that the NON CHE S2S sessions impacted their intent to get screened for CRC. Equity of access to health information and the health care system can be achieved with precision public health strategies. The $\mathrm{COE}$ bi-directional Community-to-Bench model facilitated community connections through the NON CHE and increased awareness of CRC risk reduction, screening, treatment, and research. The NON CHE combined with S2S is a powerful tool to engage communities with the greatest health care needs and positively impact an individual's intent to "get screened" for CRC.
\end{abstract}

Keywords Equity of access - Rural health · Community health educator - Cancer prevention and control · Precision public health $\cdot$ Implementation research in cancer disparities

\section{Introduction}

Rural residents continue to have higher incidence and mortality from colorectal cancer (CRC) than individuals who reside in urban areas [1-4]. Furthermore, rural residents typically experience greater disparities from most cancers $[1,4-6]$, with a larger number of racial/ethnic minorities living in areas that tend to be medically underserved [5-7]. Comparisons between rural and urban residents continue to

Michael A. Preston

prestonm2@vcu.edu

1 Department of Health Behavior and Policy, Office of Health Equity \& Disparities Research-Community Outreach \& Engagement, Virginia Commonwealth University, PO Box 980149, 830 East Main Street, Richmond, VA 23298-0149, USA highlight differences in access to preventive strategies that reduce disparities among racial/ethnical minorities and lowincome residents [1, 8-10]. Such health care inequities have also been highlighted in underserved communities during COVID-19 [11-13]. During this time, CRC screening disparities have been exacerbated and could potentially threaten decades of work to address CRC disparities in medically underserved communities [11, 12]. Individuals living in medically underserved communities such as rural communities, coupled with low socio-economic status, have lower survival rates and higher mortality rates compared to individuals at higher income levels $[5,6]$. Strategies to reduce disparities in cancer detection, treatment, and outcomes among racial/ethnic minorities and low-income residents have been gaining momentum with the introduction of the National Cancer Institute's (NCI's) National Outreach Network Community Health Educator (NON CHE) program, which 
was established to reach underserved areas through strong community partnerships [14-17]. The overall goal of the NON CHE program was to implement a long-term outreach and education plan to increase awareness about cancer risk reduction [17].

NON CHE efforts identified barriers to CRC screening and implemented Screen 2 Save (S2S), a groundbreaking $\mathrm{CRC}$ outreach and screening initiative. S2S was created in response to recommendations by the Blue Ribbon Panel for the Cancer Moonshot and endorsed by the National Cancer Advisory Board to increase CRC screening among rural, minority, and underserved areas by increasing community knowledge, awareness, and engagement activities [17].

Efforts to increase knowledge, awareness, and engagement activities among rural residents with low CRC screening adherence (including racial/ethnic minorities and lowincome residents) are needed to reduce cancer disparities [17-22] With current provisions in the ACA and the Prevention and Public Health Fund, it is critical to explore community-level opportunities that reduce disparities among high-risk communities. The COE bi-directional Communityto-Bench model facilitated community connections through NON CHE in which we assessed the impact of the S2S initiative on CRC screening knowledge and CRC screening intent in rural and underserved communities identified as CRC "Hot Spots" [23, 24].

\section{Methods}

This study used pre-intervention and post-intervention data from multiple community engagement events examining a NON CHE combined with the S2S intervention on CRC knowledge and screening intent among rural and underserved communities. Descriptive and comparative analyses were used to examine the role of the NON CHE S2S on CRC knowledge and CRC screening intent. Data included demographics, current CRC knowledge, awareness, and future $\mathrm{CRC}$ health plans. A multivariate linear regression was fit to survey scores for CRC knowledge. One-hundred and seventy surveys were collected from participants from 26 counties in the southeastern region of the Massey Cancer Center catchment area (an area identified as a CRC "Hot Spot") [23]. Surveys were self-administered among targeted communities. Each participant completed a 14-item survey before and after the intervention. Participant eligibility criteria included self-identifying as a racial or ethnic minority, being 45 years of age or older, and residing in a rural community within the MCC catchment area. All eligible participants who completed the survey received a nominal monetary gift for their time and effort. This study was approved by the Virginia Commonwealth University Institutional Review Board.

\section{Conceptual Framework}

Constructs from the Health Belief Model, Theory of Planned Behavior, Andersen's Behavioral Model of Health Services Use, and Community-to-Bench Model were integrated for this study [24, 25]. Each model provided an important concept related to CRC screening intention, sociodemographics, current CRC knowledge, and family health history for CRC. The NON CHE S2S project worked with community partners to identify CRC "Hot Spots" [23]. and determined the best approach to intervene in these areas. This approach included delivery of NCI-approved community-level education on CRC knowledge, risk factors, and screening options as a method to promote CRC screening.

\section{Measures and Statistical Analysis}

Participants answered 14 pre- and post-test questions. Each correct answer was given one point. For questions with multiple correct answers, the proportion of correct answers selected was awarded in points. Participants could score up to 14 points on this scale. Questions without a response were given a score of 0 ; however, participants who did not respond to any pre-test questions were considered to be missing data.

Demographic information collected included age, gender, Hispanic or Latino origin, race, and education. Race was expressed as separate indicator covariates for Black or White participants due to other races not having enough participants to properly estimate their effects (Asian: $n=5$, American Indian: $n=7$, Pacific Islander: $n=1$ ) and due to 2 participants marking themselves as both Black and White. Education was recorded on a scale including $8^{\text {th }}$ grade or less, some high school, high school diploma or GED, some college, college graduate, or unknown. Due to low counts $(n=1)$, data from participants with $8^{\text {th }}$ grade or lower education were combined with the unknown data to form an unknown/other category.

Summary statistics are reported in the form of means and standard deviations for continuous variables and frequencies and proportions for categorical variables. A multivariable linear regression was fit to participants' test scores with age, race, gender, and education as covariates. Statistical significance was evaluated at an alpha level of 0.05 . All statistical analysis was performed using R Statistical Software (version 3.5.2; R Foundation for Statistical Computing, Vienna, Austria).

\section{Results}

NON CHE S2S engaged 441 participants in rural and underserved communities located in VCU Massey Cancer Center's catchment area, which spans counties located in Central, Southwestern, and Eastern Virginia. Demographics 
included Black (62.9\%), American Indian (4.1\%), Asian (2.9\%), White (25.9\%), and Latino (1.8\%) men and women between the ages of 18 and 87 (Table 1). The NON CHE S2S initiative included partnerships with local faith-based organizations $(n=4)$ with a majority of the recruitment efforts from these sites. Of those engaged, 170 completed pre- and post-assessments of the NON CHE S2S and received CRC education. Results illustrated the greatest positive change in answers for questions "A stool test (FIT/FOBT) checks your stool (poop) for......" (62.6 to 73.5\%) and "In general, a colonoscopy should be performed every 10 years starting at age....." (70.1 to $87.7 \%)$.

The difference in participants' CRC knowledge had an overall mean of 0.92 , with a standard deviation of 2.56 . At baseline, White participants had significantly higher CRC knowledge scores, correctly answering $1.94(p=0.007)$ more questions on average than Black participants. After receipt of the NON CHE S2S intervention, there were no significant differences in CRC knowledge by race. A greater number of post-test questions were answered correctly with a mean of 12.45 and a standard deviation of 2.57. Female participants exhibited significantly higher CRC knowledge after controlling for the effect of other demographic variables $(p=0.004)$ (Table 2). Female study participants also had significantly greater differences between their CRC knowledge scores, exhibiting an average gain in CRC knowledge of 1.08 questions more than men $(p=0.043)$ (Table 3). Additionally, college graduates answered an average of 1.36 more questions correctly than high school graduates after controlling for the effects of other demographic characteristics $(p=0.016)$.

Table 1 Descriptive characteristics of the overall sample

\begin{tabular}{ll}
\hline Variable & $n(\%)$ \\
\hline$N$ & 170 \\
Age (mean [sd]) & $46.62(22.34)$ years \\
Gender & \\
$\quad$ Female & $n=126(74.1)$ \\
$\quad$ Unknown/other gender & $n=14(8.2)$ \\
Race/ethnicity & \\
$\quad$ American Indian & $n=7(4.1)$ \\
$\quad$ Asian & $n=5(2.9)$ \\
$\quad$ Black American & $n=107(62.9)$ \\
$\quad$ Pacific Islander & $n=1(0.6)$ \\
$\quad$ White American & $n=44(25.9)$ \\
$\quad$ Non-Hispanic & $n=152(98.1)$ \\
Education & \\
$\quad$ High school graduate & $n=27(15.9)$ \\
Some high school & $n=7(4.1)$ \\
Some college & $n=31(18.2)$ \\
College graduate & $n=89(52.4)$ \\
Unknown/other education & $n=16(9.4)$ \\
\hline
\end{tabular}

Older participants also tended to demonstrate less improvement-specifically, a 1-year increase in participant age corresponded to an adjusted average decrease in improvement of 0.02 questions. Greater than $95 \%$ of participants agreed that NON CHE S2S sessions impacted their intent to get screened for CRC.

\section{Discussion}

In light of the COVID-19 pandemic, we see inequities in the health care system being highlighted and missed opportunities to get individuals screened for CRC $[12,26]$. There were many downstream effects related to CRC screening that need to be addressed, especially in communities with the greatest health care needs. Such disparities in care continue to affect communities that are typically underserved or most vulnerable [27]. Delays in CRC screening may have "grave" health policy and practice implications. Inequities in social

Table 2 Linear model for post-test scores

\begin{tabular}{lrlr}
\hline Predictors & Estimates & 95\% CI & \multicolumn{1}{l}{$p$} \\
\hline Intercept $^{2}$ & 10.87 & 8.91 to 12.83 & $<0.001$ \\
Female $^{1}$ & 1.56 & 0.52 to 2.60 & 0.004 \\
Unknown gender $^{1}$ & 0.41 & -2.47 to 3.29 & 0.779 \\
Age & -0.02 & -0.04 to 0.00 & 0.016 \\
Some high school $^{2}$ & -0.21 & -2.19 to 1.77 & 0.832 \\
Some college $^{2}$ & 0.11 & -1.23 to 1.44 & 0.873 \\
College graduate $^{2}$ & 1.36 & 0.26 to 2.47 & 0.016 \\
Unknown/other education $^{2}$ & 1.04 & -1.22 to 3.30 & 0.366 \\
Black $_{\text {White }}$ & 0.43 & -0.97 to 1.82 & 0.546 \\
\hline
\end{tabular}

${ }^{1}$ Reference category-male

${ }^{2}$ Reference category-high school graduate

Table 3 Linear model for difference in post- and pre-test scores

\begin{tabular}{lcll}
\hline Predictors & Estimates & $95 \%$ CI & $p$ \\
\hline Intercept & 2.04 & 0.06 to 4.01 & 0.043 \\
Female $^{1}$ & 1.08 & 0.03 to 2.12 & 0.043 \\
Unknown gender $^{1}$ & 2.84 & -0.04 to 5.72 & 0.053 \\
Age & -0.02 & -0.04 to 0.00 & 0.023 \\
Some high school $^{2}$ & 1.01 & -0.97 to 2.99 & 0.315 \\
Some college $^{2}$ & -1.33 & -2.67 to 0.00 & 0.051 \\
College graduate $^{2}$ & -0.21 & -1.31 to 0.89 & 0.711 \\
Unknown/other education $^{2}$ & 0.21 & -2.26 to 2.68 & 0.869 \\
Black $^{\text {White }}$ & -0.70 & -2.15 to 0.75 & 0.341 \\
l $^{1}$ Reference category-male & -1.19 & -2.71 to 0.34 & 0.126 \\
${ }^{2}$ Reference category-high school graduate & &
\end{tabular}


determinants of health influence all health outcomes and health risks for populations most vulnerable to poor outcomes. The COVID-19 pandemic demonstrated the need to have alternative plans in place for CRC screening. So, what has been accomplished in this study "could" be adapted to other public health problems (e.g., low birth weights, breast cancer, COVID-19) [28, 29].

Community outreach and engagement interventions in rural and underserved areas should be a priority for community leaders, policy makers, and funders who wish to improve community involvement in health-related activities. The NON CHE as a community outreach and engagement resource has become a popular choice for addressing public health problems in communities where other approaches have failed [15, 16, 28, 30-36]. NON CHEs have gained momentum as a key mechanism to involve rural and underserved communities in cancer-related activities [15, 16, 35]. In its 2002 report, The Future of the Public's Health, the Institute of Medicine recommended that government public health agencies provide direct support for community health improvement initiatives by assessing community needs, providing technical assistance, and developing solutions that improve health status [37]. In spite of such efforts, cancer continues to plague underserved communities and create enormous health disparities, particularly within minority communities [37-43].

In addition to the community outreach and engagement bi-directional Community-to-Bench model, interventions such as S2S have shown significant success at increasing community knowledge, awareness, and engagement for CRC $[17,23,30,44-46]$. A combination of the NON CHE and $\mathrm{S} 2 \mathrm{~S}$ as an intervention was a powerful tool to increase CRC knowledge and future health plans such as CRC screening intent. Such activities allowed for greater engagement of rural and underserved communities with lower screening rates. To engage underserved areas, it is imperative that researchers understand the challenges that may be encountered.

Engagement of hard-to-reach communities in research involved flexibility and a multifaceted approach. Engagement activities in this study included activities with community-based organizations, faith-based organizations, and local health fairs. The COE Community-to-Bench model allowed the NON CHE to facilitate bi-directional communication with stakeholders involved with study activities [35]. Stakeholders included faith-based leaders, community-based leaders, and cancer advocates. Such activities included "Lunch and Learn," which was the most effective way to disseminate CRC information in this population. This activity provided a healthy meal to participants and offered CRC information to all participants with an opportunity for questions and answers. Another activity involved community advocates who provided education to their existing social networks as a method to increase residents' exposure to CRC messages that are designed to influence healthy screening behavior. Community advocates included cancer survivors, caregivers, and community members with an interest in reducing cancer disparities within their communities.

\section{Implications for Public Health Policy and Practice}

During a public health crisis and for health-related information in general, it is critical to provide all communities with accurate and relevant information. How different communities receive and interpret health-related information deserves further examination, especially for underserved communities. It is also important to understand community-level opportunities that reduce disparities in information asymmetry among high-risk populations. The " $80 \%$ In Every Community" is the latest national initiative by the National Colorectal Cancer Roundtable's (NCCRT) to screen for CRC. NCCRT states that achieving $80 \%$ would prevent 277,000 new cases and 203,000 deaths by 2030 [47, 48]. There are many barriers that are perceived to assist in the increased incidence of CRC groups that tend to go unscreened. The COE bi-directional Community-to-Bench model facilitated community connections through the NON CHE and S2S intervention. Thus, the COE bi-directional Community-toBench model is a community-level opportunity for the NON CHE and S2S to reduce barriers to CRC screening and to increase CRC knowledge, awareness, and screening intent for communities that need it the most. Exploration of barriers that increase disparities among medically underserved populations is critical to the work of NON CHEs.

\section{Conclusion}

Equity of access to health information and the health care system can be achieved with precision public health strategies. Our study supports the implementation of the NON $\mathrm{CHE}$ and S2S intervention in rural and underserved communities. The NON CHE S2S program facilitated community connections and increased awareness of CRC risk reduction, screening, treatment, and research. Such programs should be considered as successful mechanisms that increase the knowledge, awareness, and engagement of rural and underserved communities. The NON CHE combined with S2S is a powerful way to address health inequities, engage communities with the greatest health care needs, and impact participants' intent to "Get Screened."

Acknowledgements Special thanks to Heidi Sankala-Bauer, $\mathrm{PhD}$, Scientific Writing Manager at Massey Cancer Center, for her technical review. 
Funding This work was supported by the NCI Designated Massey Cancer Center with funding from NIH-NCI Cancer Center Support Grant P30 CA016059 and by the Virginia Tobacco Region Revitalization Commission. The funders had no role in the study design, data collection and analysis, decision to publish, or preparation of the manuscript.

\section{Declarations}

Conflict of Interest The authors declare no competing interests.

\section{References}

1. Zahnd We, James As, Jenkins Wd et al (2018) Rural-urban differences in cancer incidence and trends in the United States. Cancer Epidemiol Biomarkers Prev. 27(11):1265-1274

2. Cole Am, Jackson Je, Doescher M (2013) Colorectal cancer screening disparities for rural minorities in the United States. J Prim Care Community Health. 4(2):106-111

3. Campo S, Askelson Nm, Routsong T, Graaf Lj, Losch M, Smith H (2008) The green acres effect: the need for a new colorectal cancer screening campaign tailored to rural audiences. Health Educ Behav. 35(6):749-762

4. Preston Ma, Glover-Collins K, Ross L et al (2018) Colorectal cancer screening in rural and poor-resourced communities. Am J Surg. 216(2):245-250

5. Hines R, Markossian T, Johnson A, Dong F, Bayakly R (2014) Geographic residency status and census tract socioeconomic status as determinants of colorectal cancer outcomes. Am J Public Health 104(3):E63-E71

6. Hines Rb, Markossian Tw (2012) Differences in late-stage diagnosis, treatment, and colorectal cancer-related death between rural and urban African Americans and Whites in Georgia. J Rural Health. 28(3):296-305

7. Js Luque, Dm Tyson, Sa Bynum et al (2011) A Social network analysis approach to understand changes in a cancer disparities community partnership network. Ann Anthropol Pract. 35(2):112-135

8. Sutton A, Lichter Dt, Sassler S (2019) Rural-urban disparities in pregnancy intentions, births, and abortions among us adolescent and young women, 1995-2017. Am J Public Health. 109(12):1762-1769

9. Yeary K, Flowers E, Ford G et al (2011) Development of a community-based participatory colorectal cancer screening intervention to address disparities, Arkansas, 2008-2009. Prev Chronic Dis 8(2):A47

10 Theuer Cp, Wagner Jl, Taylor Th et al (2001) Racial and ethnic colorectal cancer patterns affect the cost-effectiveness of colorectal cancer screening in the United States. Gastroenterology. 120(4):848-856

11. Balzora S, Issaka RB, Anyane-Yeboa A, Gray DM, 2nd, May FP (2020) Impact of COVID-19 on colorectal cancer disparities and the way forward. Gastrointest Endosc. 92(4):946-950

12. Dorn Av, Cooney Re, Sabin Ml (2020) Covid-19 exacerbating inequalities in the US. Lancet (London, England). 395(10232):1243-1244

13. Laurencin CT, McClinton A (2020) The COVID-19 pandemic: a call to action to identify and address racial and ethnic disparities. Journal of Racial and Ethnic Health Disparities 7(3):398-402

14. Rodday Am, Parsons Sk, Snyder F et al (2015) Impact of patient navigation in eliminating economic disparities in cancer care. Cancer. 121(22):4025-4034

15. Curry Wj, Lengerich Ej, Kluhsman Bc et al (2011) Academic detailing to increase colorectal cancer screening by primary care practices in Appalachian Pennsylvania. Bmc Health Serv Res. 11:112

16 Paskett Ed, Cooper Mr, Stark N et al (2002) Clinical trial enrollment of rural patients with cancer. Cancer Pract. 10(1):28-35

17. Coughlin Ss, Costanza Me, Fernandez Me et al (2006) CDCfunded intervention research aimed at promoting colorectal cancer screening in communities. Cancer. 107(5 Suppl):1196-1204

18. Tsoh Jy, Tong Ek, Sy Au, Stewart Sl, Gildengorin Gl, Nguyen Tt (2018) Knowledge of colorectal cancer screening guidelines and intention to obtain screening among nonadherent Filipino, Hmong, and Korean Americans. Cancer. 124(Suppl 7):1560-1567

19. Fyffe Dc, Hudson Sv, Fagan Jk, Brown Dr (2008) Knowledge and barriers related to prostate and colorectal cancer prevention in underserved Black Men. J Natl Med Assoc. 100(10):1161-1167

20. Hamlyn S (2008) Reducing The incidence of colorectal cancer in African Americans. Gastroenterol Nurs 31(1):39-42

21 Guerra Ce, Dominguez F, Shea Ja (2005) Literacy and knowledge, attitudes, and behavior about colorectal cancer screening. J Health Commun. 10(7):651-663

22. Goldsmith G, Chiaro C (2008) Colorectal cancer screening: how to help patients comply. J Fam Pract 57(7):E2-E7

23 Siegel Rl, Sahar L, Robbins A, Jemal A (2015) Where can colorectal cancer screening interventions have the most impact? Cancer Epidemiol Biomarkers Prev. 24(8):1151-1156

24. Tossas Ky, Watson Ks, Colditz Ga, Thomas Cr, Stewart Jh, Winn $\mathrm{Ra}$ (2020) Advocating for a "Community To Bench Model" in the 21st century. Ebiomedicine. 53:102688

25 Tabbarah M, Nowalk Mp, Raymund M, Jewell Ik, Zimmerman Rk (2005) Barriers and facilitators of colon cancer screening among patients at faith-based neighborhood health centers. J Community Health. 30(1):55-74

26 Davis Tc, Arnold Cl, Rademaker Aw et al (2012) FOBT completion in FQHCS: impact of physician recommendation, FOBT information, or receipt of the FOBT kit. J Rural Health. 28(3):306-311

27. Gorodeski Ez, Goyal P, Cox Zl et al (2020) Virtual visits for care of patients with heart failure in the era of Covid-19: a statement from the Heart Failure Society of America. J Card Fail. S1071-9164(1020):30367-30365

28. Sarfaty M, Feng S (2005) Uptake of colorectal cancer screening in an uninsured population. Prev Med 41(3-4):703-706

29. Whitaker De, Snyder Fr, San Miguel-Majors S1, Bailey Lo, Springfield Sa (2020) Screen To Save: results from NCI's Colorectal Cancer Outreach and Screening Initiative to promote awareness and knowledge of colorectal cancer in racial/ethnic and rural populations. Cancer Epidemiol Biomarkers Prev. 29(5):910-917

30. Harris Jr, Brown Pk, Coughlin S et al (2005) The Cancer Prevention and Control Research Network. Prev Chronic Dis. 2(1):A21

31 Kerner Jf, Cazap E, Yach D et al (2009) Comprehensive cancer control-research \& development: knowing what we do and doing what we know. Tumori. 95(5):610-622

32 Kerner Jf, Guirguis-Blake J, Hennessy Kd et al (2005) Translating research into improved outcomes in comprehensive cancer control. Cancer Causes Control. 16(Suppl 1):27-40

33 Butterfoss Fd, Goodman Rm, Wandersman A (1996) Community coalitions for prevention and health promotion: factors predicting satisfaction, participation, and planning. Health Educ Q. 23(1):65-79

34 Fawcett Sb, Lewis Rk, Paine-Andrews A et al (1997) Evaluating community coalitions for prevention of substance abuse: the case of project freedom. Health Educ Behav. 24(6):812-828

35 Baquet $\mathrm{Cr}$ (2012) A model for bidirectional community-academic engagement (CAE): overview of partnered research, capacity enhancement, systems transformation, and public trust in research. J Health Care Poor Underserved. 23(4):1806-1824 
36. Baquet $\mathrm{Cr}$, Commiskey P (1999) Colorectal cancer epidemiology in minorities: a review. J Assoc Acad Minor Phys. 10(3):51-58

37. Institute of Medicine Committee on Assuring the Health of the Public in the 21st C. In: the future of the public's health in the 21st century. National Academies Press (US), Washington (DC). Copyright 2003 by the National Academy of Sciences. All rights reserved. 2002

38 Mullins Cd, Blatt L, Gbarayor Cm, Yang Hw, Baquet C (2005) Health disparities: a barrier to high-quality care. Am J Health Syst Pharm. 62(18):1873-1882

39. Lawsin C, Duhamel K, Weiss A, Rakowski W, Jandorf L (2007) Colorectal cancer screening among low-income African Americans in East Harlem: a theoretical approach to understanding barriers and promoters to screening. J Urban Health 84(1):32-44

40. Shokar Nk, Carlson Ca, Weller Sc (2008) Factors associated with racial/ethnic differences in colorectal cancer screening. J Am Board Fam Med. 21(5):414-426

41 Lemon Sc, Zapka Jg, Estabrook B, Benjamin E (2006) Challenges to research in urban community health centers. Am J Public Health. 96(4):626-628

42 Ayanian Jz, Zaslavsky Am, Guadagnoli E et al (2005) Patients' perceptions of quality of care for colorectal cancer by race, ethnicity, and language. J Clin Oncol. 23(27):6576-6586

43 Lasser Ke, Murillo J, Medlin E et al (2009) A multilevel intervention to promote colorectal cancer screening among community health center patients: results of a pilot study. Bmc Fam Pract. $10: 37$

44. Rutledge W, Gibson R, Siegel E et al (2006) Arkansas Special Populations Access Network perception versus reality-cancer screening in primary care clinics. Cancer 107(8 Suppl):2052-2060

45 Siegel R1, Jemal A, Wender Rc, Gansler T, Ma J, Brawley Ow (2018) An assessment of progress in cancer control. Ca Cancer J Clin. 68(5):329-339

46. Sabatino Sa, Habarta N, Baron Rc et al (2008) Interventions to increase recommendation and delivery of screening for breast, cervical, and colorectal cancers by healthcare providers systematic reviews of provider assessment and feedback and provider incentives. Am J Prev Med. 35(1 Suppl):S67-S74

47. Fedewa SA, Goodman M, Flanders WD et al (2015) Elimination of cost-sharing and receipt of screening for colorectal and breast cancer. Cancer 121(18):3272-3280

48. Meester RG, Doubeni CA, Zauber AG et al (2015) Public health impact of achieving $80 \%$ colorectal cancer screening rates in the United States by 2018. Cancer. 121(13):2281-2285

Publisher's Note Springer Nature remains neutral with regard to jurisdictional claims in published maps and institutional affiliations. 